\title{
Cellules satellites et cellules souches musculaires
}

\section{Bénédicte Chazaud}

Le muscle strié squelettique adulte normal est capable de régénérer après une lésion, recouvrant ainsi complètement sa fonctionnalité. On sait depuis plusieurs décennies que cette capacité est due aux cellules satellites logeant le long des myofibres. Au début des années 2000 , la myologie fondamentale a bénéficié du développement de nouvelles technologies et de l'émergence de l'étude des cellules souches adultes, qui ont identifié les cellules satellites comme les cellules souches adultes du muscle strié squelettique. Ces techniques ont également permis d'identifier plusieurs types de cellules souches non-satellites résidant dans le muscle et capables de former du muscle. Cet article présente une chronologie rapide des connaissances sur le sujet et aborde des questions actuelles quant à la biologie des cellules souches du muscle.

Les cellules satellites, des cellules souches uniques

Les cellules satellites sont connues depuis les années 1960. Leur découvreur, Alexander Mauro, avait prédit qu'elles pourraient participer à la reconstruction du muscle après une lésion [1], les noyaux des myofibres étant post-mitotiques. Pendant environ 40 ans, la recherche sur les cellules satellites a permis de caractériser le programme myogénique adulte lequel permet à la cellule satellite, une fois activée lors d'une lésion, de former les nouvelles myofibres (Figure 1).

Les cellules satellites peuvent être aisément extraites du muscle et cultivées. In vitro, elles reproduisent les différentes étapes de la myogenèse adulte jusqu'à la fusion en myotubes plurinucléés contractiles, et sont dotées de capacité d'auto-renouvellement. À la fin des années 90 , il a été montré in vitro que des cellules mononucléées exprimant Myf5 mais pas MyoD sont présentes à côté des myotubes bien différenciés. De plus, ces cellules sont capables de reformer une culture entière, incluant des myotubes et des cellules mononucléées, et ce pendant plusieurs passages, remplissant la définition des cellules souches à savoir des cellules capables de se différencier et de s'autorenouveler [2,3].

Bénédicte Chazaud Institut NeuroMyoGène, UMR CNRS 5310, Inserm U1217

Université Claude Bernard

Lyon 1

8 , avenue Rockfeller, 69008 Lyon, France

Contact

benedicte.chazaud@

inserm.fr
La découverte du gène Pax 7 par l'équipe de Michael Rudnicki (OHRI, Ottawa) a été une grande avancée dans la biologie des cellules satellites. Ce facteur de transcription est indispensable à la survie des cellules satellites [4]. Peter Zammit dans l'équipe de Terry Partridge (King's College, Londres) a montré en 2004, en utilisant le modèle de fibres musculaires isolées, que les cellules satellites activées entrent en prolifération et expriment à la fois Pax7 et MyoD. Les cellules qui s'engagent dans la différenciation terminale surexpriment MyoD et répriment l'expression de Pax7 alors que les cellules qui s'auto-renouvellent répriment l'expression de MyoD et surexpriment Pax7 [5]. Le fait que MyoD puisse être réprimé après avoir été pleinement exprimé par les cellules myogéniques a représenté un changement conceptuel dans la façon d'appréhender la plasticité des cellules myogéniques au cours de la myogenèse adulte. L'année suivante, la même équipe londonienne a fait la démonstration in vivo chez la souris que les cellules satellites pouvaient à la fois participer à la régénération musculaire en se différenciant en myofibres, et s'auto-renouveler pour reformer le stock de cellules satellites disponibles pour une régénération ultérieure [6]. Les chercheurs du King's College ont greffé une seule myofibre provenant d'une souris dont les noyaux sont repérables par un gène rapporteur sous le contrôle de Myf5. Ils ont pu ainsi démontrer la participation des sept noyaux de la myofibre donneuse aux deux processus de formation de nouvelles fibres et de nouvelles cellules satellites, établissant ainsi que les cellules satellites sont les cellules souches adultes du muscle strié squelettique adulte. Ces travaux ont été récemment confirmés par trois laboratoires indépendants montrant que la déplétion (par la technologie Cre-Lox) des cellules exprimant Pax7 (donc les cellules satellites) empêche complètement la régénération musculaire après une lésion massive chez la souris [7-9]. Depuis ces travaux, les équipes de recherche s'attachent maintenant à comprendre plus en détail la biologie des cellules satellites, et en particulier s'il 


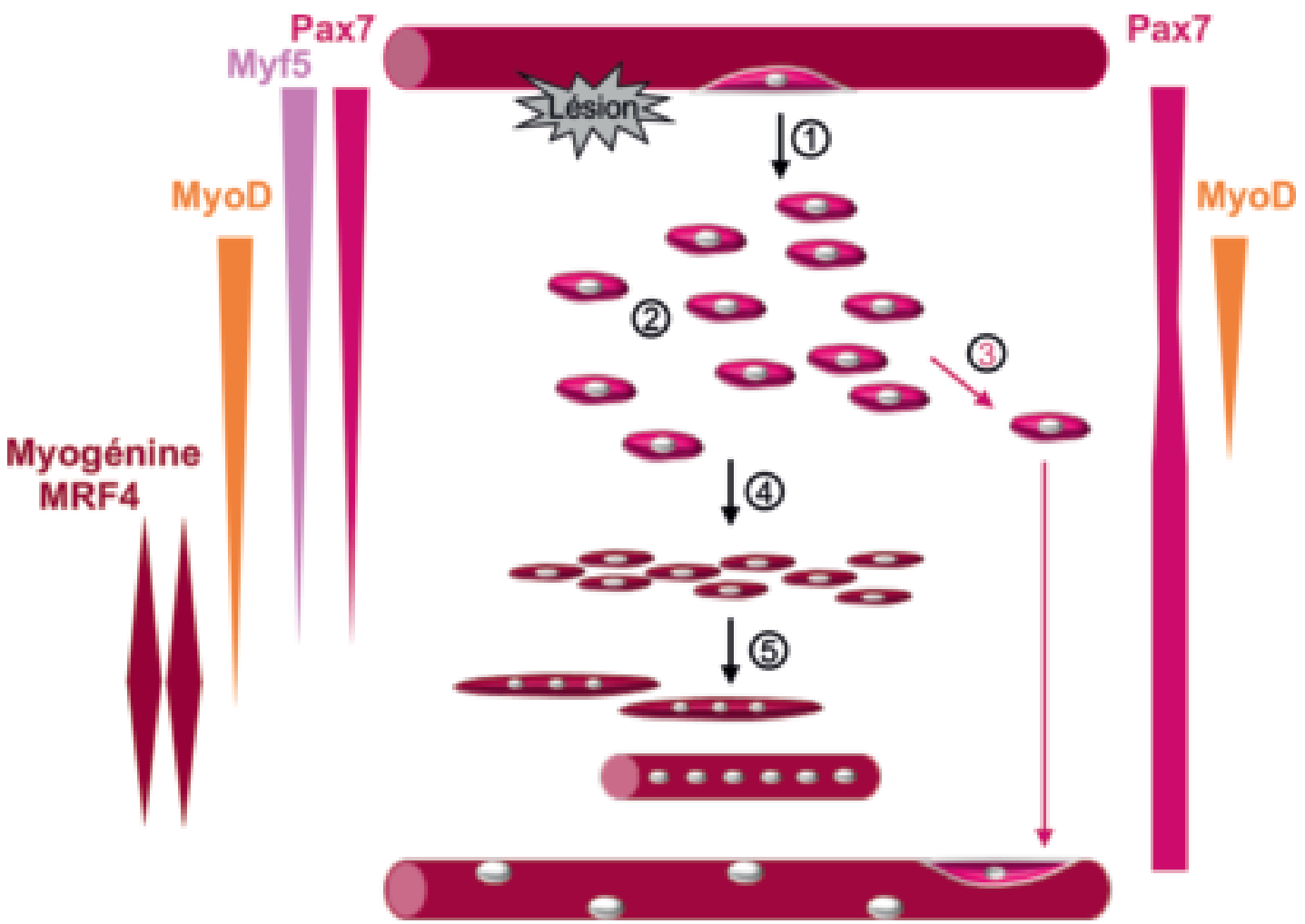

Figure 1

Myogenèse adulte post-lésionnelle. Après une lésion, les cellules satellites sortent de quiescence et s'activent (1). Les précurseurs myogéniques (ou myoblastes) prolifèrent pendant la phase d'expansion (2). Une petite partie des cellules suit le programme d'auto-renouvellement en sortant du cycle cellulaire et en entrant en quiescence pour reformer le stock des cellules satellites (3). La grande majorité des cellules s'engage dans le programme de différenciation myogénique terminal (sortie du cycle cellulaire et expression du programme myogénique) (4). Ensuite, elles fusionnent pour former les nouvelles myofibres (5). Le niveau d'expression des facteurs de transcription myogéniques et de Pax7 est indiqué pour chaque étape.

existe une hiérarchie entre différentes populations de cellules satellites. L'équipe de Michael Rudnicki a montré à l'aide d'outils de traçage génétique que les cellules satellites exprimant Pax7 et n'exprimant pas Myf5 sont plus en amont dans la hiérarchie des cellules satellites, c'est-à-dire qu'elles sont capables de plus d'auto-renouvellement par rapport aux cellules exprimant les deux facteurs de transcription qui s'engagent davantage dans la différenciation myogénique et la régénération musculaire. L'autorenouvellement des cellules souches a d'ailleurs été décrit comme asymétrique, produisant une cellule souche et une cellule fille, seule cette dernière s'engageant dans la progénie musculaire [10]. Ce concept, démontré à l'aide du modèle de la fibre isolée, reste à valider in vivo.

L'équipe de Shahragim Tajbakhsh, à l'institut Pasteur, a également montré in vivo que les cellules satellites n'expriment pas Pax7 de façon homogène. Des expériences de greffes sérielles chez l'animal combinées à des marquages du cycle cellulaire ont montré que les cellules satellites qui expriment fortement Pax7 sont en amont dans la hiérarchie des cellules souches et sont plus quiescentes. Elles donnent naissance aux cellules exprimant plus faiblement Pax7 qui sont plus engagées dans la régénération du muscle [11].

À ce jour, les cellules satellites peuvent être qualifiées de cellules souches adultes monopotentes car elles ne peuvent se différencier qu'en myocytes. Les équipes de recherche impliquées dans la biologie des cellules satellites s'intéressent désormais aux mécanismes moléculaires qui contrôlent les différentes étapes de la vie d'une cellule satellite (activation, prolifération, différenciation, fusion, auto-renouvellement) dans des conditions physiologiques et pathologiques variées. On peut citer par exemple les travaux récents de l'équipe de Pura Muñoz-Cánoves qui montrent des défauts dans la protéostasie (équilibre des protéines dans la cellule) des cellules souches du sujet très âgé aboutissant à un défaut de régénération [12]. 


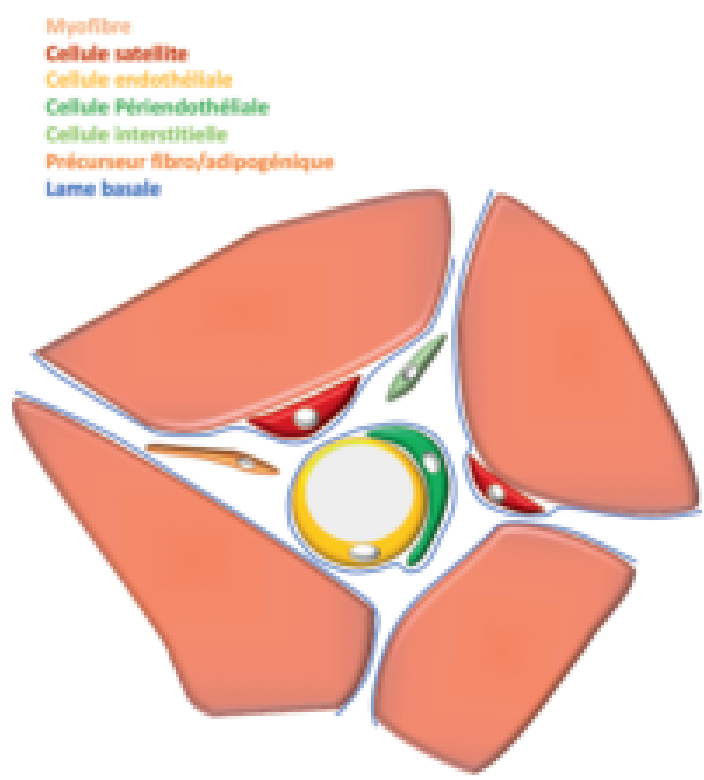

Figure 2

Cellules satellites et cellules souches musculaires. Les cellules satellites sont logées sous la lame basale qui entoure la myofibre, et sont préférentiellement localisées à proximité des vaisseaux sanguins. Le tissu musculaire est très dense et l'espace interstitiel, c'est-à-dire entre les myofibres, est ténu. On y trouve cependant les capillaires sanguins, composés de cellules endothéliales et de cellules périendothéliales dont certaines présentent des propriétés de cellules souches myogéniques. Différentes populations de cellules interstitielles ont été identifiées comme ayant des propriétés de cellules souches myogéniques. Cependant, les relations entre cellules souches musculaires et cellules satellites sont encore mal comprises. Enfin, dans ce même espace confiné se trouvent les cellules précurseurs fibro-adipogéniques, dont l'ontogénie est parfaitement distincte de celle de la lignée myogénique : elles sont capables de se différencier uniquement en fibroblastes et en adipocytes dans le muscle strié squelettique.

\section{Les cellules souches adultes du muscle strié squelettique}

Entre les myofibres se trouve un espace interstitiel ténu au sein duquel cohabitent de nombreux types cellulaires dont les cellules vasculaires et périvasculaires, les terminaisons nerveuses, des cellules interstitielles plus ou moins bien définies, et quelques cellules de l'immunité dans des conditions normales. Avant que les études de délétion des cellules exprimant Pax7 précitées montrent que l'absence des cellules satellites empêche toute régénération musculaire, de nombreuses équipes de recherche ont cherché à démontrer l'existence de cellules souches interstitielles capables de réaliser un programme myogénique, et ce en vue de les utiliser notamment dans des programmes de thérapie cellulaire dans les pathologies musculaires dégénératives. Un grand nombre de populations cellulaires a été décrit, notamment après isolement prospectif des cellules du muscle (c'est-à-dire selon des marqueurs que les cellules expriment à leur surface). Ces cellules sont capables de former des myotubes in vivo et de participer à la régénération musculaire in vivo quand on les injecte dans le muscle. Peu d'études ont passé l'étape de l'efficacité préclinique du gros animal et de la voie d'injection systémique. Citons ici deux populations cellulaires, isolées selon des méthodes non prospectives mais par des techniques de culture cellulaire. Ces cellules sont capables de rejoindre le muscle lésé lorsqu'elles sont injectées par voie artérielle et de participer à la régénération musculaire dans des modèles de dystrophie. D'une part, les cellules MuStem, du laboratoire nantais Oniris, qui ont montré une efficacité thérapeutique avec amélioration de fonction chez le chien GRMD (modèle de la myopathie de Duchenne) en étant capables de faire exprimer la dystrophine dans des myofibres et de se trouver également en position satellite [13]. D'autre part, les mésangioblastes, identifiés par l'équipe de Giulio Cossu, à l'Institut San Raffaele de Milan, qui sont également capables de participer à la régénération musculaire et qui ont fait l'objet d'un essai clinique de phase I-Ila [14].

\section{Conclusion}

La relation entre les cellules souches musculaires interstitielles et les cellules satellites (Figure 2) reste une énigme à ce jour. Les premières sont capables de former du muscle in vitro ou in vivo après une étape in vitro, et sont pourtant incapables de suppléer à la disparition des secondes après une blessure massive. Il faudra sans doute affiner les modèles d'étude, en particulier les modèles lésionnels chez la souris. De nouvelles technologies somme le CyTOF permettront d'affiner nos connaissances sur les populations cellulaires en présence dans le muscle adulte et d'en déduire des interactions et/ou des hiérarchies. Cette technique permet d'étudier individuellement les cellules selon une cinquantaine de marqueurs qu'elles expriment à leur surface, qualitativement et quantitativement. Nul doute que de nouvelles sous-populations satellites et non-satellites seront identifiées dans un futur proche. Leurs propriétés pourront être étudiées grâce aux outils toujours plus efficaces de délétion génique dans un type cellulaire donné, et à un moment donné (délétion spécifique et inductible). On pourra ainsi distinguer les populations ayant réellement des propriétés myogéniques intrinsèques des cellules qui offrent un environnement propice aux cellules myogéniques pour permettre la régénération musculaire. En effet, la recherche sur les cellules souches intéresse également les pathologies musculaires. Des travaux récents de l'équipe de Michael Rudnicki ont montré que les cellules satellites déficientes pour la dystrophine montrent une altération dans le processus d'auto-renouvellement [15]. Si 
l'impact de ce phénomène n'a pas encore été évalué et si la myopathie de Duchenne reste en premier lieu une pathologie touchant la myofibre, des altérations de l'homéostasie des cellules satellites participent probablement à l'aggravation de la maladie.

Satellite cells and muscle stem cells

\section{LIENS D'INTÉR̂̂T}

L'auteur déclare n'avoir aucun lien d'intérêt concernant les données publiées dans cet article.

\section{RÉFÉRENCES}

1. Mauro A. Satellite cell of skeletal muscle fibers. $J$ Biophys Biochem Cytol 1961; 9 : 493-5.

2. Yoshida N, Yoshida S, Koishi K, et al. Cell heterogeneity upon myogenic differentiation: down-regulation of MyoD and Myf-5 generates reserve cells. J Cell Sci 1998; 111 : 769-79.

3. Friday BB and Pavlath GK. A calcineurin- and NFAT-dependent pathway regulates Myf5 gene expression in skeletal muscle reserve cells. $J$ Cell Sci $2001 ; 114$ : 303-10.

4. Seale P, Sabourin LA, Girgis-Gabardo A. et al. Pax7 is required for the specification of myogenic satellite cells. Cell $2000 ; 102$ : 777-86.

5. Zammit PS, Golding JP, Nagata Y, et al. Muscle satellite cells adopt divergent fates: a mechanism for self-renewal? J Cell Biol $2004 ; 166: 347-57$
6. Collins CA, Olsen I, Zammit PS, et al. Stem cell function, selfrenewal, and behavioral heterogeneity of cells from the adult muscle satellite cell niche. Cell 2005; $122: 289-301$.

7. Lepper C, Partridge TA, Fan CM. An absolute requirement for Pax7-positive satellite cells in acute injury-induced skeletal muscle regeneration. Development $2011 ; 138$ : 3639-46.

8. Murphy MM, Lawson JA, Mathew SJ, et al. Satellite cells, connective tissue fibroblasts and their interactions are crucial for muscle regeneration. Development 2011; 138 : 3625-37.

9. Sambasivan R, Yao R, Kissenpfennig A, et al. Pax7-expressing satellite cells are indispensable for adult skeletal muscle regeneration. Development 2011; 138 : 3647-56.

10. Kuang S, Kuroda K, Le Grand F, Rudnicki MA. Asymmetric self-renewal and commitment of satellite stem cells in muscle. Cell 2007; 129: 999-1010.

11. Rocheteau P, Gayraud-Morel B, Siegl-Cachedenier I, et al. A subpopulation of adult skeletal muscle stem cells retains all template DNA strands after cell division. Cell $2012 ; 148$ : 112-25.

12. Garcia-Prat L, Martinez-Vicente M, Perdiguero E, et al. Autophagy maintains stemness by preventing senescence. Nature 2016 ; 529 : 37-42

13. Rouger K, Larcher T, Dubreil L, et al. Systemic delivery of allogenic muscle stem cells induces long-term muscle repair and clinical efficacy in Duchenne muscular dystrophy dogs. Am $J$ Pathol 2011; 179 : 2501-18.

14. Cossu G, Previtali SC, Napolitano S, et al. Intra-arterial transplantation of HLA-matched donor mesoangioblasts in Duchenne muscular dystrophy. EMBO Mol Med 2015 ; 7 : 1513-28.

15. Dumont NA, Wang YX, von Maltzahn J, et al. Dystrophin expression in muscle stem cells regulates their polarity and asymmetric division. Nat Med 2015 ; 21 : 1455-63.

\title{
sfm
}

\section{Bulletin d'adhésion 2018}

\author{
NOM/Prénom \\ $\square$ Clinique \\ $\square$ Fondamentale \\ - Autre fonction \\ Adresse : \\ Code Postal : \\ Ville : \\ E-mall : \\ ADHÉSION : le desire adhérer en qualité de (raver la mention inutile) \\ Membre titulaire : $40 \mathrm{C}$ \\ Membre étudiant : gratuit (fournir un justificatif de votre qualite ơ etudiant non salarie) \\ RËGLEMENT \\ - Je joins un chique lbelle a Fordre de la Societte Française de Mrologie d'un montant de $40 \mathrm{C}$ \\ 口 J'effectue un virement bancaire de $40 \mathrm{C}$ (RIB de la SFM à demander à Rémi Mounier) \\ A RETOURNER A : \\ remi.mouniereunivityon 1.fr \\ Retmi MOUNIER - Trésorier de la SFM \\ CR HDR CNRS - UMR CNRS 5534 \\ Centre de Génétique et de Physiologle Moléculaire et Cellulaire \\ Universite Claude Bernard tyon 1 \\ Batiment Gregor Mendel - 2è étage \\ 16 Rue Raphati Dubois \\ F.69622 Villeurbanne Cedex
}

N.B. : Bulletin à photocopier et à diffuser à toute personne intéressée 